AAS 12-250

\title{
SPACECRAFT DEBRIS AVOIDANCE USING POSITIVELY INVARIANT CONSTRAINT ADMISSIBLE SETS
}

\author{
Morgan Baldwin, Avishai Weiss $\nmid$ llya Kolmanovsky; R. Scott Erwin ${ }^{\S}$
}

\begin{abstract}
To cope with the growing amount of debris in Earth orbit, spacecraft collision avoidance capabilities are necessary. In this paper, we propose an approach to debris avoidance maneuvering based on the use of safe positively invariant sets in order to steer the spacecraft, under closed-loop control, around a piece of debris. A connectivity graph of forced equilibria is computed based on the overlap of these invariant sets, and a graph search algorithm is then implmented in order to find the shortest path around the debris. Fast growth distance computation is employed for on-board real-time applicability. Simulation results are presented that illustrate this approach.
\end{abstract}

\section{INTRODUCTION}

More than 22,000 identifiable objects, mostly debris, are being tracked today in space and many of them represent spacecraft collision risk. Motion planning problems with debris avoidance have been studied in robotics applications ${ }^{1,2}$ for many years. Related problems in spacecraft applications have several unique features. Firstly, the environment is relatively uncluttered as compared to typical robotics applications. This gives an opportunity to perform fuel efficient debris avoidance maneuvers. The minimization of fuel consumption and maneuver efficiency are very important in spacecraft applications. Excessive use of fuel can shorten spacecraft mission life, even if the debris collisions are avoided. Secondly, the spacecraft dynamics and orbital motions are quite different from those of typical robots while the control authority of spacecraft thrusters is very limited. Thirdly, the position of the debris and the states of the spacecraft are estimated but may not be accurately known. Fourth, fast computational algorithms are necessary for deployment within the control units of small satellites. Fifth, spacecraft may be flying alone or in formation and may need to avoid multiple debris that may be moving relative to the spacecraft. These unique features lead to both challenges and opportunities in the development of specific and effective maneuvering solutions for spacecraft applications.

The interest in spacecraft trajectory optimization with obstacle avoidance has increased in recent years. In Ref. 3, this problem was formulated as an optimal control problem with path constraints constructed as 'keep out' zones to avoid obstacles. The SOCS software was then used to solve the problem. An optimal control problem formulation was used in Ref. 4 to solve the minimumfuel rendezvous between a chief and deputy vehicle. Collision avoidance constraints were added to the problem in the form of inequality constraints, resulting in an inequality constrained, nonlinear

\footnotetext{
* Research Aerospace Engineer, Space Vehicles Directorate, Air Force Research Laboratory, Albuquerque, NM.

${ }^{\dagger}$ Graduate Assistant, Department of Aerospace Engineering, The University of Michigan, Ann Arbor, MI.

${ }^{\ddagger}$ Professor, Department of Aerospace Engineering, The University of Michigan, Ann Arbor, MI.

${ }^{\S}$ Principal Research Aerospace Engineer, Space Vehicles Directorate, Air Force Research Laboratory, Albuquerque, NM.
} 
programming problem. The method involved solving a sequence of unconstrained optimal control problems, whose solution converges to the solution of the original problem. Maneuver avoidance strategies have also been defined utilizing collision avoidance probabilities. Ref. 5 presents collision avoidance strategies based upon the number of evasive maneuvers, expected risk reduction, false alarm rate, and required propellant consumption and mass fraction for an accepted collision probability.

Artificial potential function guidance is used in Ref. 6 and 7 to determine a rendezvous solution on a path free of obstacles. A potential function is developed with the intent that a minimum occurs at a desired relative position and then a dynamic control law is used to ensure the trajectory is obstacle free. ${ }^{6}$ A 3-D static optimization over final relative position and time-of-flight such that obstacles are avoided and cost is optimized is presented in Ref. 7. Feedback is incorporated by re-planning over either constant or variable time intervals.

The spacecraft obstacle avoidance problem has also been treated using linear programming techniques. ${ }^{8-11}$ In Ref. 8 , the minimum-fuel avoidance maneuver problem is formulated with linear constraints and discrete dynamics modeled as a linear, time-varying system. The trajectory optimization problem is formulated as a linear programming problem with the capability of including operational constraints and the optimal number of maneuvers is determined in Ref. 9. A mixedinteger linear program results from combining collision avoidance, trajectory optimization, and fleet assignment to obtain the optimal solution for spacecraft maneuvers as shown in Ref. 10. A robust linear programming technique is proposed in Ref. 11. The maneuver can be constructed by solving a linear programming problem with no integer constraints and guaranting collision avoidance with respect to bounded navigation uncertainty.

Our approach in this paper is based on the use of constraint-admissible positively invariant sets ${ }^{12}$ centered around forced and unforced spacecraft equilibria in the Hill-Clohessy-Wiltshire or HCW (relative motion) frame, ${ }^{13}$ and corresponding to different choices of the Linear Quadratic (LQ) control gain. The finite set of these equilibria used for constructing debris avoidance maneuvers is referred to as a virtual net. Given an estimate of the debris position, we build on-line a connectivity graph that identifies the equilibria in the virtual net between which the spacecraft can move, with guaranteed collision-free and thrust-limited motions. The construction of the connectivity graph is based on fast growth distance computation between two ellipsoidal-type sets. We then employ real-time graph search algorithms to determine an efficient path between the equilibria and control gains used that permit debris avoidance. Simulation results are reported that illustrate the approach.

In our approach we do not rely on precise assignment of spacecraft position to the time instants along the trajectory but wait for the appropriate conditions to switch to the next set-point and controller gain. This approach is conservative and may facilitate fault-tolerant and disturbance-tolerant execution of the maneuvers.

\section{RELATIVE MOTION MODEL}

In the traditional treatment of the relative motion problems, the spacecraft motion is considered relative to the (non-inertial) Hill's frame with the origin at a target location on the nominal orbit. For generic elliptic orbits, the linearization of the nonlinear equations of motion equations yields a set of linear time-varying Tschauner-Hempel (TH) equations. For circular orbits, these TH equations reduce to the well known linear time-invariant HCW equations. See Ref. 13. A nominal circular orbit is assumed in this work. 


\section{Nonlinear equations of motion}

The relative position vector of the spacecraft with respect to a target location on a circular orbit is expressed as

$$
\delta \vec{r}=x \hat{\imath}+y \hat{\imath}+z \hat{k}
$$

where $x, y$ and $z$ are the components of the position vector of the spacecraft relative to the target location and $\hat{\imath}, \hat{\jmath}, \hat{k}$ are the unit vectors of the Hill's frame. The Hill's frame has its $x$-axis along the orbital radius, $y$-axis along the orbital track, and $z$-axis orthogonal to orbital plane. The position vector of the spacecraft with respect to the center of the Earth can be expressed as $\vec{R}=\vec{R}_{0}+\delta \vec{r}=$ $\left(R_{0}+x\right) \hat{\imath}+y \hat{\jmath}+z \hat{k}$, where $R_{0}$ is the nominal orbital radius. The nonlinear equations of motion for the spacecraft (relative to an inertial frame) can be expressed in vector form as

$$
\ddot{\vec{R}}=-\mu \frac{\vec{R}}{R^{3}}+\frac{1}{m_{c}} \vec{F}
$$

where $\vec{F}$ is the vector of external forces applied to the spacecraft, $R=|\vec{R}|, m_{c}$ is the mass of the spacecraft, $\mu$ is the gravitational constant, and

$$
\ddot{\vec{R}}=\left(\ddot{x}-2 n \dot{y}-3 n^{2} x\right) \hat{\imath}+(\ddot{y}+2 n \dot{x}) \hat{\jmath}+\left(\ddot{z}+n^{2} z\right) \hat{k} \text {. }
$$

In these equations, $n=\sqrt{\frac{\mu}{R_{0}^{3}}}$ denotes the mean motion of the nominal orbit. Similar equations can be used to describe the motion of the debris.

\section{Linearized HCW equations in discrete-time}

For $\delta r<<R$, the linearized HCW equations ${ }^{13}$ approximate the relative motion of the spacecraft on a circular orbit as

$$
\begin{gathered}
\ddot{x}-3 n^{2} x-2 n \dot{y}=\frac{F_{x}}{m_{c}}, \\
\ddot{y}+2 n \dot{x}=\frac{F_{y}}{m_{c}}, \\
\ddot{z}+n^{2} z=\frac{F_{z}}{m_{c}},
\end{gathered}
$$

where $F_{x}, F_{y}, F_{z}$ are components of the external force vector (excluding gravity) acting on the spacecraft.

Assuming a sampling period of $\Delta T \mathrm{sec}$, we can convert the model (2) to a discrete-time form

$$
X(t+1)=A X(t)+B U(t)
$$

where $X(t)=[x(t), y(t), z(t), \dot{x}(t), \dot{y}(t), \dot{z}(t)]^{\mathrm{T}}$ is the state vector at the time instant $t \in Z^{+}$, $U(t)=\left[F_{x}(t), F_{y}(t), F_{z}(t)\right]^{\mathrm{T}}$ is the control vector of thrust forces at the time instant $t \in Z^{+}$, and $A, B$ are the discretized matrices obtained from $\mathrm{HCW}$ equations. Alternatively, the control vector $U$ can represent an instantaneous change in the velocity of the spacecraft, $\Delta v$, induced by thrust, with an appropriately re-defined $B$-matrix. 


\section{DEBRIS AVOIDANCE BASED ON A VIRTUAL NET}

In this paper we treat a spacecraft motion planning problem with debris avoidance. We initially assume that the debris is stationary relative to the spacecraft. The case of moving debris will be discussed later in the paper.

Our approach to debris avoidance is based on utilizing constraint-admissible positively invariant sets $^{12,14}$ centered around the spacecraft forced and unforced equilibria. A finite set of these equilibria used for constructing debris avoidance maneuvers is referred to as a virtual net. Given an estimate of the debris position, we build a connectivity graph that identifies the equilibria in the virtual net between which the spacecraft can move, with guaranteed collision-free motion and within the available thrust authority. We then employ graph search to determine an efficient path between the equilibria that permits debris avoidance.

\section{Virtual Net}

The virtual net comprises a finite set of equilibria, $X_{e}(r)$, corresponding to a finite set of prescribed spacecraft positions $r \in \mathcal{N}=\left\{r_{1}, r_{2}, \ldots, r_{n}\right\} \subset R^{3}$,

$$
X_{e}\left(r_{k}\right)=\left[\begin{array}{c}
r_{k} \\
0
\end{array}\right]=\left[\begin{array}{c}
r_{k x} \\
r_{k y} \\
r_{k z} \\
0 \\
0 \\
0
\end{array}\right], k=1, \cdots, n
$$

See Figure 1. We assume that for all $r \in \mathcal{N}$, the corresponding values of control necessary to support the specified equilibria in steady-state satisfy the imposed thrust limits.

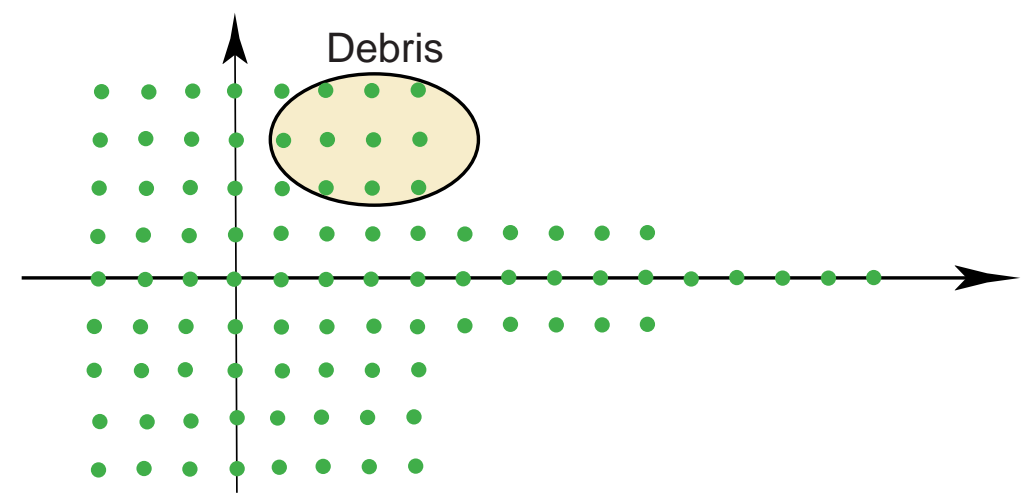

Figure 1: The virtual net for debris avoidance.

\section{LQ Controller with Gain Switching}

A conventional Linear-Quadratic (LQ) feedback is used to control the spacecraft to a commanded equilibrium in (4),

$$
U=K\left(X-X_{e}(r)\right)+\Gamma r=K X+H(K) r
$$


where

$$
\begin{gathered}
\Gamma=\left[\begin{array}{ccc}
-3 n^{2} m_{c} & 0 & 0 \\
0 & 0 & 0 \\
0 & 0 & n^{2} m_{c}
\end{array}\right], \\
H(K)=\Gamma-K\left[\begin{array}{l}
I_{3} \\
0_{3}
\end{array}\right],
\end{gathered}
$$

and where $I_{3}$ denotes the $3 \times 3$ identity matrix and $0_{3}$ denotes the $3 \times 3$ zero matrix. This LQ controller provides an asymptotically stable closed-loop system but does not enforce the constraints.

To provide greater flexibility in handling constraints, a multimode controller architecture is employed. ${ }^{14}$ Specifically, we assume that a finite set of LQ gains $K \in \mathcal{K}=\left\{K_{1}, \cdots, K_{m}\right\}$ is available to control the spacecraft. By using a large control weight in the LQ cost functional, motions with low fuel consumption yet large excursions can be generated; using a large control weight in the LQ cost, motions with short transition time can be generated. ${ }^{15}$ We assume that a preference ordering has been defined and the gains are arranged in the order of descending preference, from $K_{1}$ being the highest preference gain to $K_{m}$ being the lowest preference gain.

\section{Positively Invariant Sets}

The ellipsoidal set

$$
\bar{C}(r, K)=\left\{X \in R^{6}: \frac{1}{2}\left(X-X_{e}(r)\right)^{T} P(K)\left(X-X_{e}(r)\right) \leq 1\right\} \subset R^{6},
$$

where $(A+B K)^{T} P(A+B K)-P<0, P=P(K)>0$, is positively invariant. Positive invariance implies that any trajectory of the closed-loop system that starts in $\bar{C}(r, K)$ is guaranteed to stay in $\bar{C}(r, K)$ as long as the same LQ gain $K$ is used and the set-point command $r$ is maintained. To achieve the positive invariance, the matrix $P$ can be the solution of the discrete-time Riccati equation or the above Lyapunov equation for the closed-loop asymptotically stable system. We note that because the system is linear, the positive invariance of $\bar{C}(r, K)$ implies the positive invariance of the scaled set

$$
C(r, K, \rho)=\left\{X \in R^{6}: \frac{1}{2}\left(X-X_{e}(r)\right)^{T} P(K)\left(X-X_{e}(r)\right) \leq \rho^{2}\right\}, \quad \rho \geq 0
$$

Geometrically, the set $C(r, K, \rho)$ corresponds to an ellipsoid scaled by the value of $\rho$ and centered around $X_{e}(r), r \in \mathcal{N}$.

\section{Debris Representation}

We use a set, $O(z, Q)$, centered around the position $z \in R^{3}$, to over-bound the position of the debris, i.e.,

$$
O(z, Q)=\left\{X \in R^{6}:(S X-z)^{T} Q(S X-z) \leq 1\right\}, Q=Q^{T}>0
$$

where

$$
S=\left[\begin{array}{llllll}
1 & 0 & 0 & 0 & 0 & 0 \\
0 & 1 & 0 & 0 & 0 & 0 \\
0 & 0 & 1 & 0 & 0 & 0
\end{array}\right]
$$

This set can account for the debris and spacecraft physical sizes, and also for the uncertainties in the estimation of the debris/spacecraft position. Note that the set $O(z, Q)$ has an ellipsoidal shape 
in the position directions and it has a cylindrical shape in the velocity directions. Ellipsoidal sets rather than polyhedral sets are used to over-bound the debris since ellipsoidal bounds are typically produced by position estimation algorithms, such as the Extended Kalman Filter (EKF).

\section{Debris Avoidance Approach}

Consider now $r_{i} \in \mathcal{N}$, representing a possible position on the net that the spacecraft can move to as a part of the obstacle avoidance maneuver. Suppose that the current state of the spacecraft is $X\left(t_{0}\right)$ at the time instant $t_{0} \in Z^{+}$. If there exists a $\rho \geq 0$ and $K_{j} \in \mathcal{K}$ such that

$$
X\left(t_{0}\right) \in C\left(r_{i}, K_{j}, \rho\right) \text { and } O(z, Q) \cap C\left(r_{i}, K_{j}, \rho\right)=\emptyset,
$$

the spacecraft can move to the position $r_{i} \in \mathcal{N}$ by engaging the control law with $r(t)=r_{i}$ and $K(t)=K_{j}, t \geq t_{0}$, and without hitting the debris. This idea underlies our subsequent approach to debris avoidance, where we maintain the spacecraft trajectories within the tube formed by positively invariant sets that do not overlap with the debris.

\section{Growth Distances}

The minimum value of $\rho \geq 0$ for which $O(z, Q) \bigcap C(r, K, \rho) \neq \emptyset$ is referred to as the growth distance, similarly to Ref. 16. This growth distance can also be viewed as the least upper bound on the values of $\rho$ for which $O(z, Q)$ and $C(r, K, \rho)$ do not intersect. See Figure 2. We use the notation $\rho_{g}(r, K, Q, z)$ to reflect the dependence of the growth distance on the set-point $r \in \mathcal{N}$, the control gain $K \in \mathcal{K}$ and the obstacle parameters $Q$ and $z$.

Note that the growth distance depends on the position of the debris which may be unknown in advance. Consequently, the growth distance computations have to be performed online.

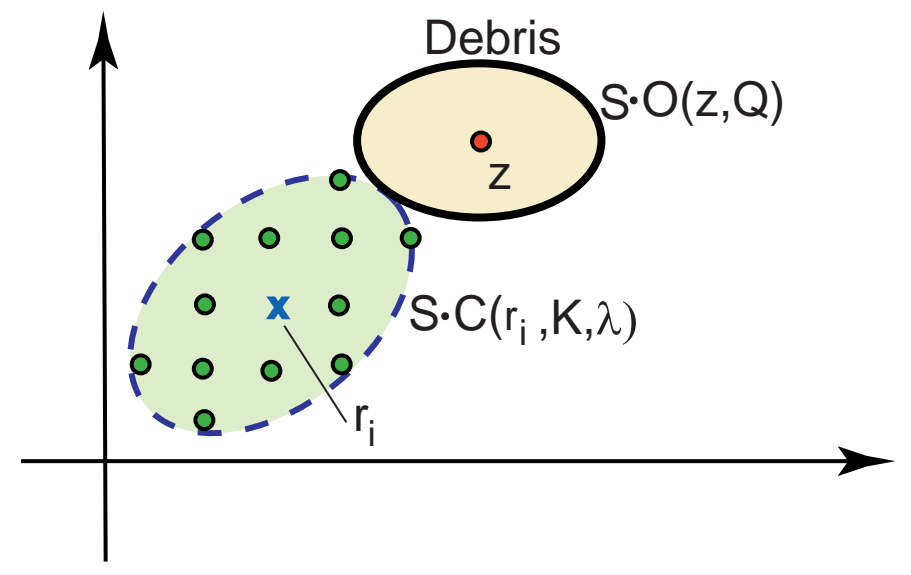

Figure 2: The positively invariant set is grown till touching the debris. The spacecraft can move from any of the equilibria on the virtual net inside the positively invariant set $C(r, K, \rho)$ to $X_{e}\left(r_{i}\right)$ marked by ' $x$ ' without colliding with the debris.

Since the spacecraft maneuvers have to be performed using limited thrust, we additionally define a maximum value of $\rho=\rho_{u}(r, K)$ for which $X \in C\left(r, K, \rho_{u}(r, K)\right)$ implies that the thrust $U=$ $K X+H(K) r$ satisfies the imposed thrust limits. We refer to $\rho_{u}$ as the thrust limit on growth distance. Unlike $\rho_{g}$, the value of $\rho_{u}$ does not depend on the position or shape of the obstacle and can be pre-computed off-line. Suppose that the thrust limits are expressed in the form $\|L U\| \leq 1$ 
for an appropriately defined matrix $L$ and norm $\|\cdot\|$. The computational procedures to determine $\rho_{u}(r, K)$ involves solving a bilevel optimization problem where $\|L(K X+H(K) r)\|$ is maximized subject to the constraint $X \in C(r, K, \alpha)$ and bisections are performed on the value of $\alpha$ so that the maximum value is driven to 1 .

Finally, we define the thrust limited growth distance

$$
\rho^{*}(r, K, Q, z)=\min \left\{\rho_{g}(r, K, Q, z), \rho_{u}(r, K)\right\} .
$$

Note that $X\left(t_{0}\right) \in C\left(r_{i}, K_{j}, \rho^{*}\left(r_{i}, K_{j}, z\right)\right)$ implies that the ensuing closed-loop spacecraft trajectory under the control (5) with $r(t)=r_{i}$ and $K(t)=K_{j}$ for $t \geq t_{0}$ satisfies the thrust limits and avoids debris collisions.

\section{Connectivity Graph and Graph Search}

We now introduce a notion of connectivity between two vertices of the virtual net, $r_{i} \in \mathcal{N}$ and $r_{j} \in \mathcal{N}$. The vertex $r_{i}$ is connected to the vertex $r_{j}$ if there exists a gain $K \in \mathcal{K}$ such that

$$
X_{e}\left(r_{i}\right) \in \operatorname{int} C\left(r_{j}, K, \rho^{*}\left(r_{j}, K, z\right)\right) .
$$

The connectivity implies that a spacecraft located close to an equilibrium corresponding to $r_{i}$ can transition to an equilibrium $X_{e}\left(r_{j}\right)$ by using limited thrust and avoiding collision with the debris. We note that if $r_{i}$ is connected to $r_{j}$ this does not imply that, in turn, $r_{j}$ is connected to $r_{i}$. We also note that connectivity depends on the existence of an appropriate control gain from the set of gains $\mathcal{K}$ but the condition (12) does not need to hold for all gains.

The on-line motion planning with debris avoidance is performed according to the following procedure:

Step 1: Determine the debris location and shape (i.e., $z$ and $Q$ ).

Step 2: By using fast growth distance computations, determine thrust limited growth distance based on (11), with $\rho_{g}$ computed online and $\rho_{u}$ pre-computed off-line.

Step 3: Construct a graph connectivity matrix between all $r_{i}, r_{j} \in \mathcal{N}$. In the graph connectivity matrix, if two vertices are not connected, the corresponding matrix element is zero; if they are connected the corresponding matrix element is 1 . In parallel, build the control gain selectivity matrix, which identifies the index of the highest preference gain $K$ for which $r_{i}$ and $r_{j}$ are connected. This gain will be applied if the edge connecting $r_{i}$ and $r_{j}$ is traversed.

Step 4: Perform graph search to determine a sequence of connected vertices $r[k] \in \mathcal{N}$ and control gains $K[k] \in \mathcal{K}, k=1, \cdots, l_{p}$, such that $r[1]$ satisfies the initial constraints, $r\left[l_{p}\right]$ satisfies the final constraints, and the path length $l_{p}$ is minimized.

Per the above algorithm, a graph search is utilized to determine the minimum number of equilibrium hops around a piece of debris. After the path has been determined as a sequence of the set-points and the corresponding control gains, the execution of the path proceeds by checking if the current state, $X(t)$ is in the safe positively invariant set corresponding to the next reference $r^{+}$ and next control gain $K^{+}$in the sequence; if it is, then the controller switches to this reference and control gain:

$$
X(t) \in C\left(r^{+}, K^{+}, \rho^{*}\left(r^{+}, K^{+}, z\right)\right) \rightarrow r(t)=r^{+}, K(t)=K^{+} .
$$




\section{Growth Distance Computations}

The fast online growth distance computation can greatly facilitate the implementation of the proposed approach.

For given $z \in R^{3}, r \in R^{3}$ and $K \in \mathcal{K}$, the growth distance is the minimum value of $\rho \geq 0$ for which $O(z) \cap C(r, K, \rho) \neq \emptyset$. To determine the growth distance, one enlarges $C(r, K, \rho)$ by changing $\rho$ until touching $O(z)$. Since $C(r, K, \rho)$ is compact and $O(z)$ is closed, it can be shown that the growth distance exists and is well-defined.

Define $\bar{X}=X-X_{e}(r)$ and $\alpha=2 \rho^{2}$. The problem of determining the growth distance reduces to the following constrained optimization problem:

$$
\begin{array}{ll}
\min _{\alpha, \bar{X}} & \alpha \\
\text { subject to } & \bar{X}^{\mathrm{T}} P \bar{X} \leq \alpha \\
& \left(S\left(\bar{X}+X_{e}(r)\right)-z\right)^{\mathrm{T}} Q\left(S\left(\bar{X}+X_{e}(r)\right)-z\right) \leq 1 .
\end{array}
$$

To solve this optimization problem, we use the Karush-Kuhn-Tucker (KKT) conditions. Note that the standard linear independence constraint qualification conditions hold given that $P>0$. We define

$$
\begin{aligned}
\mathcal{L} & =\alpha+\lambda_{1}\left(\bar{X}^{\mathrm{T}} P \bar{X}-\alpha\right) \\
& +\lambda_{2}\left(\left(S\left(\bar{X}+X_{e}(r)\right)-z\right)^{\mathrm{T}} Q\left(S\left(\bar{X}+X_{e}(r)\right)-z\right)-1\right),
\end{aligned}
$$

where $\lambda_{1}$ and $\lambda_{2}$ are Lagrange multipliers. The stationarity of the Lagrangian (setting partial derivative equal to zero) with respect to $\alpha$ leads to the condition $\lambda_{1}=1$. The stationarity of the Lagrangian with respect to $\bar{X}$ leads to

$$
P \bar{X}+\lambda_{2} S^{\mathrm{T}} Q S \bar{X}+\lambda_{2} S^{\mathrm{T}} Q\left(S X_{e}(r)-z\right)=0,
$$

or

$$
\bar{X}=\bar{X}\left(\lambda_{2}, r, z\right)=-\left(P+\lambda_{2} S^{\mathrm{T}} Q S\right)^{-1} S^{\mathrm{T}} Q\left(S X_{e}(r)-z\right) \lambda_{2},
$$

where $\lambda_{2} \geq 0$ is a scalar to be determined. Note that $P>0, S^{\mathrm{T}} Q S \geq 0, \lambda_{2} \geq 0$ (as the Lagrange multiplier corresponding to an inequality constraint) imply that $\left(P+\lambda_{2} S^{\mathrm{T}} Q S\right)$ is invertible.

A computational procedure to determine the growth distance can now be defined based on the bisections approach. Specifically, the bisections can be performed to find a nonnegative scalar $\lambda_{2}$ which is the root of the equation

$$
F\left(\lambda_{2}, r, z\right)=(S X-z)^{\mathrm{T}} Q(S X-z)-1=0,
$$

where

$$
X=\bar{X}\left(\lambda_{2}, r, z\right)+X_{e}(r) .
$$

Computationally, these bisections to find $\lambda_{2}$ can be performed rapidly.

An alternative procedure to using bisections is to apply a Newton-Raphson method to determine the unknown $\lambda_{2}$ in the nonlinear equation (15). Since the computations of the growth distance need to be performed for different $r \in \mathcal{N}$ and possibly $z$ (in the case the debris is moving), we 
propose an even faster approach than bisections to the distance computation based on viewing the above root finding problem as a functional root-finding problem where the parameters are $z$ and $r$. Unlike conventional root finding problem, in a functional root finding problem, a root needs to be determined as a function of a parameter.

Consider an iterative update procedure for $\lambda_{2}$ in response to changes in $z$ and $r$. Let the superscript $k$ denote the $k$ th iteration. As the Newton-Raphson's approach is based on setting a first order approximation of $F$ to zero, we consider the following approximate equation

$$
\begin{aligned}
F\left(\lambda_{2}^{k+1}, z^{k+1}, r^{k+1}\right) & \approx F\left(\lambda_{2}^{k}, z^{k}, r^{k}\right)+\frac{\partial F}{\partial \lambda_{2}}\left(\lambda_{2}^{k}, z^{k}, r^{k}\right)\left(\lambda_{2}^{k+1}-\lambda_{2}^{k}\right) \\
& +\frac{\partial F}{\partial z}\left(\lambda_{2}^{k}, z^{k}, r^{k}\right)\left(z^{k+1}-z^{k}\right)+\frac{\partial F}{\partial r}\left(\lambda_{2}^{k}, z^{k}, r^{k}\right)\left(r^{k+1}-r^{k}\right)=0 .
\end{aligned}
$$

Solving this equation leads to a predictor-corrector update algorithm of the form,

$$
\begin{aligned}
\lambda_{2}^{k+1} & =\lambda_{2}^{k}+\left\{\frac{\partial F}{\partial \lambda_{2}}\left(\lambda_{2}^{k}, z^{k}, r^{k}\right)\right\}^{-1}\left\{-F\left(\lambda_{2}^{k}, z^{k}, r^{k}\right)\right. \\
& \left.-\frac{\partial F}{\partial z}\left(\lambda_{2}^{k}, z^{k}, r^{k}\right)\left(z^{k+1}-z^{k}\right)-\frac{\partial F}{\partial r}\left(\lambda_{2}^{k}, z^{k}, r^{k}\right)\left(r^{k+1}-r^{k}\right)\right\} .
\end{aligned}
$$

The computational procedure involves first setting $r^{k}$ and $z^{k}$ to nominal values and letting several algorithm iterations to take place. Given quadratic convergence rate associated with the NewtonRaphson's method, we expect fast convergence during these initial iterations to occur. We then start varying $r^{k}$ and $z^{k}$, letting $\lambda_{2}^{k}$ respond based on (16) and letting $\bar{X}\left(\lambda_{2}^{k}, r^{k}, z^{k}\right)$, computed using (14), track the minimizer. Under suitable assumptions, and following steps of showing quadratic convergence rate for Newton's method, it can be demonstrated that the minimizer tracking accuracy, $\left\|\bar{X}^{k}-\bar{X}^{* k}\right\|$, is of order $O(\epsilon)$, where $\epsilon$ bounds $\left\|r^{k+1}-r^{k}\right\|$ and $\left\|z^{k+1}-z^{k}\right\|$, and where $\bar{X}^{* k}$ denotes the true minimizer. Consequently, slowing changes in $r$ and $z$ reduces the minimizer tracking error. To implement the algorithm, we take advantage of the explicitly known functional form for $F$, and use the following expressions

$$
\begin{gathered}
\frac{\partial \bar{X}}{\partial \lambda_{2}}=\left(P+\lambda_{2} S^{\mathrm{T}} Q S\right)^{-1}\left\{-S^{\mathrm{T}} Q\left(S X_{e}(r)-z\right)-S^{\mathrm{T}} Q S \bar{X}\right\} \\
\frac{\partial F}{\partial \lambda_{2}}=2(S \bar{X}-z+r)^{\mathrm{T}} Q\left(S \frac{\partial \bar{X}}{\partial \lambda_{2}}\right) \\
\frac{\partial \bar{X}}{\partial r}=\left(P+\lambda_{2} S^{\mathrm{T}} Q S\right)^{-1}\left\{-S^{\mathrm{T}} Q S \Omega\right\} \lambda_{2} \\
\frac{\partial F}{\partial r}=2(S \bar{X}-z+r)^{\mathrm{T}} Q\left(S \frac{\partial \bar{X}}{\partial r}+I_{3}\right) \\
\frac{\partial \bar{X}}{\partial z}=\left(P+\lambda_{2} S^{\mathrm{T}} Q S\right)^{-1} S^{\mathrm{T}} Q S \Omega \lambda_{2} \\
\frac{\partial F}{\partial z}=2(S \bar{X}-z+r)^{\mathrm{T}} Q\left(S \frac{\partial \bar{X}}{\partial z}-I_{3}\right)
\end{gathered}
$$

In these equations,

$$
X_{e}(r)=\Omega r, \text { where } \Omega=\left[\begin{array}{c}
I_{3} \\
0
\end{array}\right],
$$


and $I_{3}$ denotes the $3 \times 3$ identity matrix.

Figure 3 illustrates the growth distance tracking in the case when $z=[0.3,0.4,0.5]^{\mathrm{T}}, Q=100 I_{3}$ and $P=P^{\mathrm{T}}>0$ is determined as a solution to the Lyapunov equation. For the first 20 iterations, $r^{k}$ is a constant and then starts to vary slowly through the virtual net. The growth distance tracking is accurate. Figure 4 illustrates the growth distance tracking in the case when $r^{k}$ varies through points in the virtual net in such a way that the distance becomes zero for some of the points.

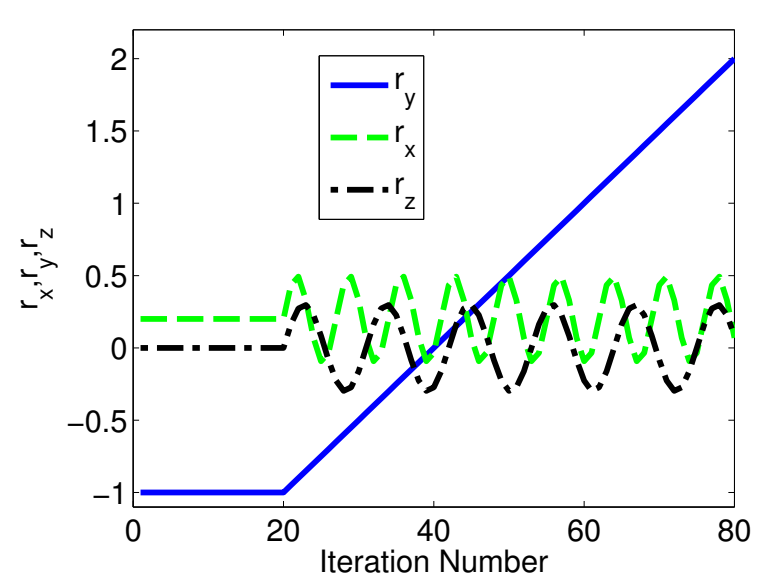

(a) Update of $r$

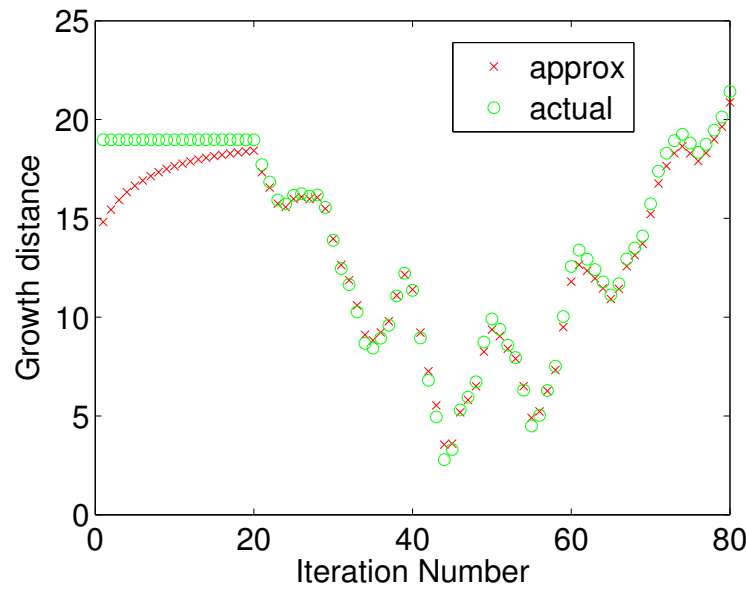

(b) Minimizer tracking

Figure 3: Implementation of parameter-dependent Newton-Raphson algorithm.

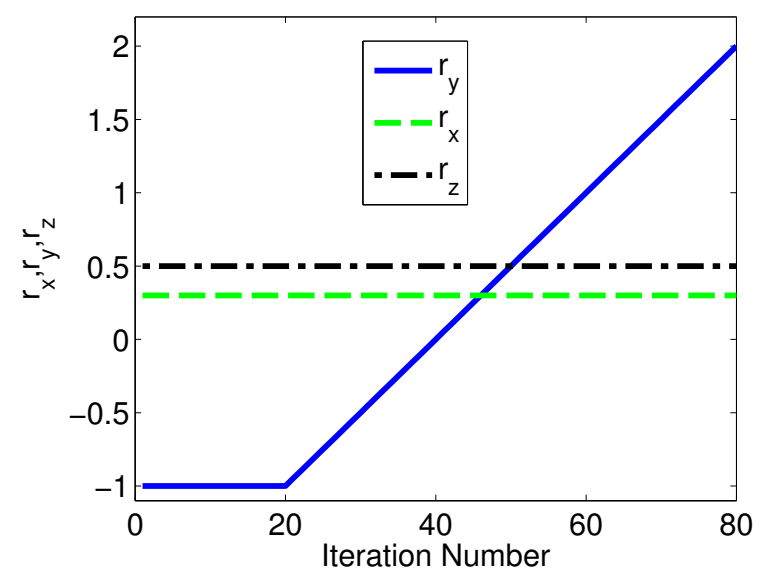

(a) Update of $r$

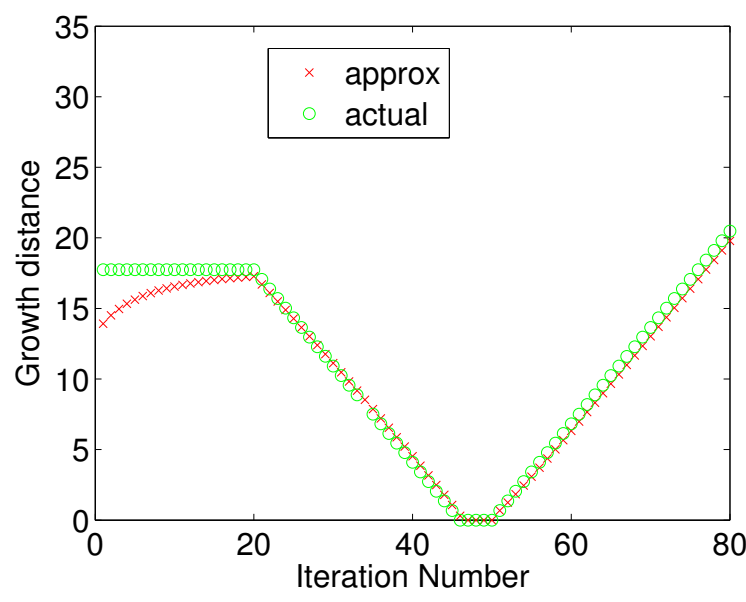

(b) Minimizer tracking

Figure 4: Implementation of parameter-dependent Newton-Raphson algorithm.

\section{Extensions}

The above approach considered a single debris which remained stationary relative to the spacecraft. Multiple stationary or moving debris can be handled with a similar approach. The basic idea 
is discussed in this paper and will be developed further in future publications. In this approach a union of a finite number of sets of ellipsoidal shape,

$$
\mathcal{D}=\bigcup_{i=1}^{i=n_{d}} O\left(z_{i}, Q_{i}\right),
$$

where the $i$ th set is denoted by $z_{i} \in R^{3}$ and the $i$ th set shape is defined by $Q_{i}=Q_{i}^{\mathrm{T}}>0$ is used to cover the predicted paths of one or multiple debris. The growth distance is redefined as the minimum distance to each of $O\left(z_{i}, Q_{i}\right), i=1, \cdots, n_{d}$, and used to construct the connectivity graph and perform motion planning. A simulation example of avoiding collision with two debris will be presented in the next section.

Another extension is to incorporate bounds on the disturbances due to air drag, solar pressure or thrust errors. The main modifications required are in the definition of the vertex connectivity and in the notion of the invariance of $C\left(r, K, \rho^{*}(r, K, z)\right)$. Specifically, we consider the vertex $r_{i}$ as being connected to the vertex $r_{j}$ if there exists $K_{j} \in \mathcal{K}$ such that for all $K \in \mathcal{K}$

$$
F_{\infty}\left(r_{i}, K\right) \subset \operatorname{int} C\left(r_{j}, K_{j}, \rho^{*}\left(r_{j}, K, z\right)\right)
$$

where $F_{\infty}\left(r_{i}, K\right)$ denotes the minimum invariant set ${ }^{14}$ of the closed-loop dynamics with the gain $K$ and set-point, $r_{i}$. This minimum invariant set plays a role similar to $X_{e}(r)$, i.e., it is the minimum asymptotic attractor of the closed loop trajectories under the influence of all possible disturbance inputs. The set $C\left(r_{j}, K_{j}, \rho^{*}\left(r_{j}, K, z\right)\right)$ must be positively invariant under the influence of disturbances satisfying the specified bounds. This in effect imposes a lower limit on $\rho^{*}\left(r_{j}, K, z\right)$ and makes the case $C\left(r_{j}, K_{j}, \rho^{*}\left(r_{j}, K, z\right)\right)=\emptyset$ more common. However, under suitable probabilistic assumptions on the independence of the disturbances for different time instants, the connectivity condition (18) can be replaced by the weaker, "disturbance-free" condition (12). See Ref. 14 for the basic approach in the multimode controller case.

\section{SIMULATION}

Simulations are now provided that illustrate our debris avoidance approach. We consider a nominal circular orbit of $850 \mathrm{~km}$ and discretize the $\mathrm{HCW}$ equations with a sampling period, $\Delta T$, of 120 seconds. We construct an approximately $2 \mathrm{~km}$ cubed virtual net and define two sets of preference ordering for our LQ gains. We refer to the first preference ordering of LQ gains as minimum fuel ordering where gains corresponding to higher control penalty and lower fuel consumption are preferred. For this ordering, we let $Q=\operatorname{diag}(100,100,100,0.1,0.1,0.1)$ and $R=\rho I_{3}$, where $\rho=2 \times 10^{9}, 2 \times 10^{8}, 2 \times 10^{7}, 2 \times 10^{6}$. We refer to the second preference ordering of LQ gains as the minimum time ordering where gains corresponding to lower control penalty and faster response are preferred. For this ordering, we keep the same $Q$ and let $R=\rho I_{3}$, where $\rho=2 \times 10^{4}, 2 \times 10^{3}, 2 \times 10^{2}, 2 \times 10^{1}$.

An ellipsoidal set over-bounds a piece of debris, $O\left(z_{1}, Q_{1}\right)$, centered at $z_{1}=\left[\begin{array}{lll}0.3 & 0.4 & 0.5\end{array}\right]^{\mathrm{T}}$ $\mathrm{km}$ and with $Q_{1}=100 I_{3}$. We use the fast distance computation technique based on bisections to determine the growth distance to the debris from each node in the net. The spacecraft's initial condition is $X(0)=X_{e}\left(r_{0}\right)$, where $r_{0}=\left[\begin{array}{llll}1.58 & 1.0 & 1.58\end{array}\right]^{\mathrm{T}}$. The target equilibrium node is $X_{e}(0)$. Finally, we do not consider thrust constraints in this simple example. Dijkstra's algorithm is used to find the shortest cost path from initial node to final node. 
Figure 5 shows the path the spacecraft takes under closed-loop control in order to avoid the debris. The case when minimum time gain preference ordering is used is shown in Figure 5a, and the case when minimum fuel gain preference ordering is used is shown in Figure 5b. In both cases, the spacecraft is able to complete the desired maneuver while successfully avoiding the debris.

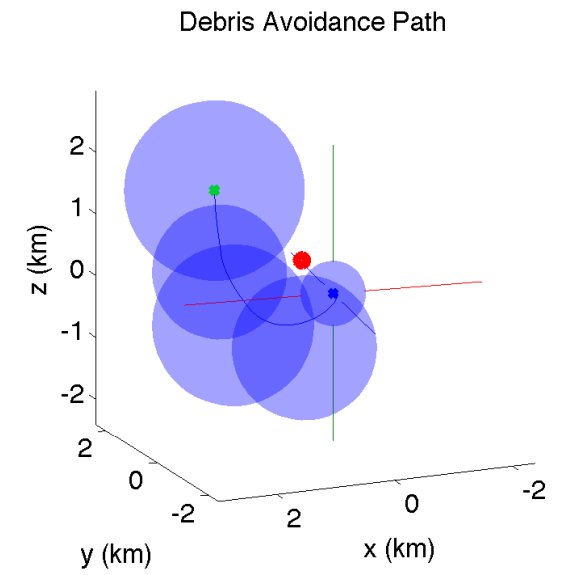

(a) Minimum time LQ gain ordering preference.

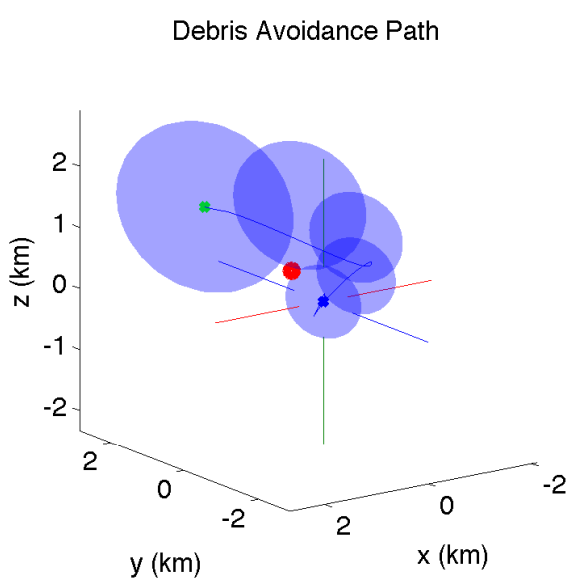

(b) Minimum fuel LQ gain ordering preference.

Figure 5: Debris Avoidance Path. The green x marks the initial node, the blue $\mathrm{x}$ marks the final node. The red ellipsoid represents the debris. The blue line is the path the spacecraft takes in order to avoid the debris. The blue ellipsoids represent the invariant sets along the path.

In Figure 6 we rerun the above simulation for a grid of initial conditions. The minimum time maneuvering, shown in Figure 6a, clearly demonstrates the initial conditions for which the manuever path is purturbed from that which it would have taken had there been no debris.

Finally, we add a second piece of debris, $O\left(z_{2}, Q_{2}\right)$, centered at $z_{2}=\left[\begin{array}{lll}-0.3 & 0.4 & 0.5\end{array}\right]^{\mathrm{T}}$ and with $Q_{2}=100 I_{3}$. In calculating the growth distance, we take the minimum distance to each of $O\left(z_{i}, Q_{i}\right)$, $i=1,2$. Figure 7 shows the path the spacecraft takes under closed-loop control in order to avoid the debris. The case of minimum time gain preference ordering is shown in Figure 7a. The case of minimum fuel gain preference ordering is shown in Figure 7b. Note that while the "minimum time" maneuver path is not noticeably changed as compared to the case with one piece of debris, the "minimum fuel" maneuver path is quite different, as the second piece of debris lies along the path the controller would have selected had the second piece of debris not been there.

\section{CONCLUSION}

The paper proposed an approach to spacecraft relative motion control which satisfies constraints on thrust and avoids collisions with debris. The approach is based on switching between set-points in a virtual net and maintaining the spacecraft trajectory within a tube formed by safe positively invariant sets. The invariant sets have ellipsoidal shape, and fast online distance computation has been proposed to determine a distance between two ellipsoidal objects.

Several extensions of this approach are of interest, including less conservative treatment of the case of multiple debris, and will be pursued in future publications. 


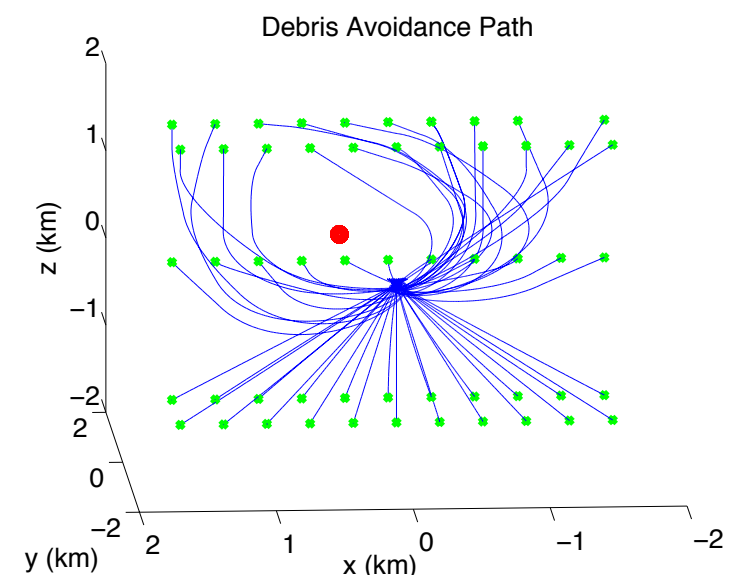

(a) Minimum time LQ gain ordering preference.

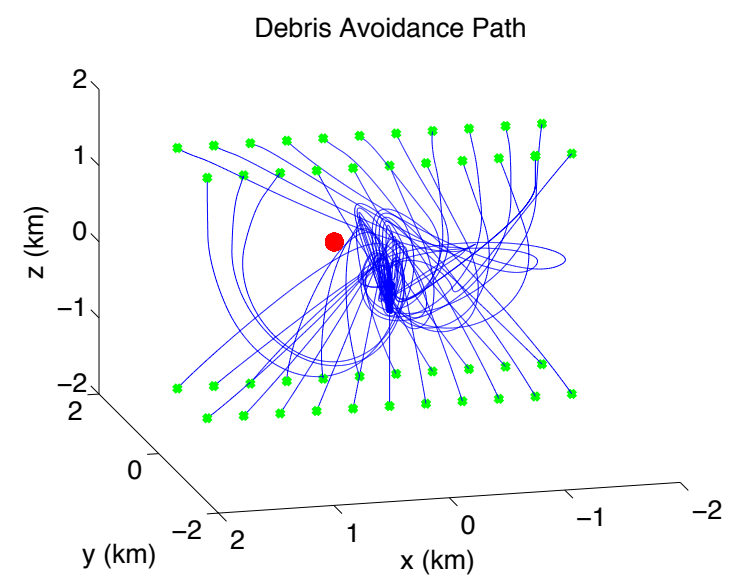

(b) Minimum fuel LQ gain ordering preference.

Figure 6: Debris Avoidance Path for many initial conditions. The green $\mathrm{x}$ marks the intial node, the blue $\mathrm{x}$ marks the final node. The red ellipsoid represents the debris. The blue line is the path the spacecraft takes in order to avoid the debris. We do not show the invariant set ellipsoids for visual clarity.

Debris Avoidance Path

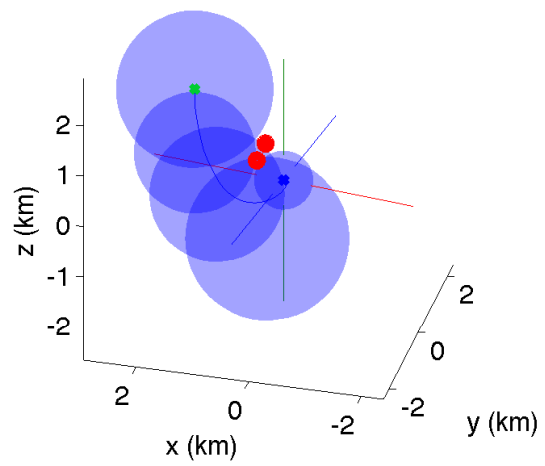

(a) Minimum time LQ gain ordering preference.
Debris Avoidance Path

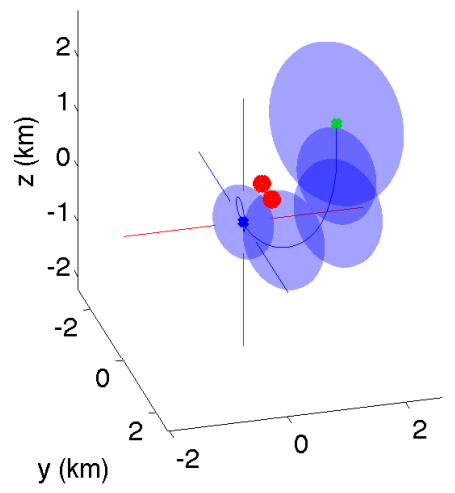

(b) Minimum fuel LQ gain ordering preference.

Figure 7: Debris Avoidance Path for 2 pieces of debris. The green $x$ marks the intial node, the blue $\mathrm{x}$ marks the final node. The red ellipsoid represents the debris. The blue line is the path the spacecraft takes in order to avoid the debris. The blue ellipsoids represent the invariant sets along the path. 


\section{REFERENCES}

[1] LaValle, S.M., Planning Algorithms, Cambridge University Press, 1996.

[2] Maia, M.H. and Galvao, R.K.H., "On The Use of Mixed-Integer Linear Predictive Control with Avoidance Constraints," International Journal of Robust and Nonlinear Control, Vol. 19, pp. 822-828.

[3] Ranieri, C"Path-Constrained Trajectory Optimization for Proximity Operations," AIAA/AAS Astrodynamics Specialist Conference, Honolulu, HI, August 2008.

[4] Epenoy, R., "Fuel Optimization for Continuous-Thrust Orbital Rendezvous with Collision Avoidance Constraint," Journal of Guidance, Control, and Dynamics, Vol. 34, No. 2, March-April 2011.

[5] Sanchez-Ortiz, N., Bello-Mora, M., and Klinkrad, H., "Collision avoidance manoeuvres during spacecraft mission lifetime: Risk reduction and required $\Delta$ V," Advances in Space Research, Vol. 38, 2006.

[6] Martinson, N., Munoz, J., and Wiens, G., "A new method of guidance control for autonomous rendezvous in a cluttered space environment," AIAA Guidance, Navigation, and Control Conference, Hilton Head, SC, August 2007.

[7] Martinson, N.,"Obstacle avoidance guidance and control algorithms for spacecraft maneuvers," AIAA Guidance, Navigation, and Control Conference, Chicago, IL, August 2009.

[8] Mueller, J., Griesemer, P., and Thomas, S., "Avoidance Maneuver Planning Incorporating StationKeeping Constraints and Automatic Relaxation,” AIAA Infotech@Aerospace 2010, Atlanta, GA, April 2010.

[9] Ulybyshev, Y., “Trajectory Optimization for Spacecraft Proximity Operations with Constraints," AIAA Guidance, Navigation, and Control Conference, Portland, OR, August 2011.

[10] Richards, A., Schouwenaars, T., How, J., and Feron, E., "Spacecraft Trajectory Planning with Avoidance Constraints Using Mixed-Integer Linear Programming,"AIAA Journal of Guidance, Control, and Dynamics, Vol. 25, No. 4, August 2002.

[11] Mueller, J., “Onboard Planning of Collision Avoidance Maneuvers Using Robust Optimization,” AIAA Infotech@Aerospace 2009, Seattle, WA, April 2009.

[12] Kolmanovsky I.V., Gilbert E.G., "Theory and Computation of Disturbance Invariant Sets for DiscreteTime Linear Systems," Mathematical Problems in Engineering, Vol. 4(4), pp. 317-367, 1998.

[13] Wie, B., Spacecraft Dynamics and Control, 2nd Ed. Reston, VA: AIAA,2010.

[14] Kolmanovsky, I.V., and Gilbert, E., "Multimode Regulators for Systems with State and Control Constraints and Disturbance Inputs," Proceedings of Workshop "Control Using Logic-Based Switching," pp. 104-117, published in an edited volume by Springer-Verlag, Editor: Morse A.S, Block Island, Rhode Island, Fall, 1997.

[15] Park, H., Di Cairano, S., and Kolmanovsky, I.V., "Linear Quadratic Model Predictive Control Approach to Spacecraft Rendezvous and Docking," Proceedings of 21st AAS/AIAA Space Flight Mechanics Meeting, New Orleans, Louisiana, USA, Paper AAS-142, 2011.

[16] Ong C.J., Gilbert E.G., "Growth Distances: New Measures for Object Separation and Penetration," IEEE Transactions on Robotics and Automation, Vol. 12(6), pp. 888-903, 1996 